Children's Hospital, Melbourne, Australia. Of children identified with LD, more than half had BP diagnosed by DSM-III-R criteria. Those with mathematics difficulties were at greater risk of BP than children who had spelling problems (69\% cf $31 \%$ affected). Children with a single LD (spelling or math) were equally likely to have an internalizing diagnosis (anxious-fearful), while those with both types of LD were more prone to externalizing disorders (hostile-aggressive or hyperactive-distractible). Of children identified with LD at 7 to 8 years, $80 \%$ had persisting literacy difficulties and $57 \%$ had arithmetic problems at 12 years of age. (Prior M, Smart D, Sanson A, Oberklaid F. Relationships between learning difficulties and psychological problems in preadolescent children from a longitudinal sample. I Am Acad Child Adolesc Psychiatry April 1999;38:429-436). (Respond: Professor Margot Prior PhD, Department of Psychology, Royal Children's Hospital, Parkville, Victoria, Australia 3052).

COMMENT. Children with learning disorders are at high risk of developing behavior problems, and children with LDs and BPs have persistence of difficulties through grade school years, particularly for boys. Mathematical LD predisposes to anxiety disorders whereas multiple LDs are predictive of hyperactivity or hostileaggressive behavior.

Cocaine exposure and behavioral problems. Children of drug abusers (CDAs) are at increased risk of internalizing and externalizing problems, attention deficits, and impulsivity. Stanger $C$ and associates, University of Vermont, found that CDAs showed more behavior problems than matched nonreferred children, but significantly less psychopathology than a sample of children referred for mental health services (I Am Acad Child Adolesc Psychiatry April 1999;38:421-428).

\title{
CORTICAL DYSFUNCTION IN AUTISM
}

Cerebellar and cortical systems in 26 high-functioning adolescents and young adults with autism were examined and compared to 26 healthy controls at the University of Pittsburgh School of Medicine. Reflexive, visually guided (cerebellar modulated) saccadic eye movements were normal whereas volitional (neocortical) saccade tasks (oculomotor delayed-response and antisaccade) were abnormal. The results demonstrate neocortical, not cerebellar, dysfunction in autism, suggesting deficits in higher cognitive mechanisms and impairments in spatial working memory and executive control over reflexive behavior. (Minshew NJ, Luna B, Sweeney JA. Oculomotor evidence for neocortical systems but not cerebellar dysfunction in autism. Neurology March 1999;52:917-922). (Reprints: Dr Nancy J Minshew, University of Pittsburgh School of Medicine, 3811 O'Hara St, 430 Bellefield Towers, Pittsburgh, PA 15213).

COMMENT. Both cerebellar and neocortical dysfunctions have been proposed as a neurologic basis for autism. Dysfunction of cerebellar vermal lobules VI and VII should be associated with dysmetric saccadic eye movements. In the present study, cerebellar-mediated saccades were normal but neocortical control of oculomotor eye movements was impaired. The findings support neuropsychological evidence of deficits in cortical, voluntary regulation of attentional focus in autism.

A new hypothesis for autism is proposed by DeLong GR, Duke University Medical Center (Neurology March 1999;52:911-916). Autism occurs in two forms: 1) Secondary to early infantile, bilateral brain damage (eg. epileptic medial temporal sclerosis, herpes simplex encephalitis, infantile spasms, tuberous 
sclerosis, congenital rubella); 2) idiopathic autism, with regression in the second year, prominent affective symptoms, and a more favorable prognosis. Idiopathic autism is characterized by a left-hemisphere serotonin deficiency, and is benefited by selective serotonin reuptake inhibitors (eg fluoxetine). Secondary autism caused by bilateral hemisphere damage has a poor prognosis, whereas idiopathic autism with a learning and memory deficit due to single hemisphere damage is compatible with higher functioning levels.

Brain weight in autism. The serotonin deficiency in autism, by permitting excessive branching of thalamocortical axons, may result in postnatal brain enlargement. Megalencephaly has been reported in some autopsied cases of autism, but a brief report of brain weights of 21 postmortem cases found the majority were normal, one micrencephalic, and only 3(15\%) were megalencephalic (Courchesne E, Muller R-A, Saitoh O. Brain weight in autism: Normal in the majority of cases, megalencephalic in rare cases. Neurology March 1999;52:1057-1059).

Polydipsia in autism. The incidence of polydipsia in 49 autistic children was higher than in retarded children studied at the Noto Second Hospital, Ishikawa, Japan. (Terai $\mathrm{K}$ et al. Excessive water drinking behavior in autism. Brain Dev 1999;21:103-106). This finding suggests a hypothalamic-pituitary dysfunction in autism.

Placental insufficiency as a risk factor for early onset schizophrenia. Hultman CM et al, University of Uppsala, Sweden, report the association of small size for gestational age and bleeding during pregnancy as risk factors for early onset schizophrenia and affective psychosis in males. (BMI 13 February 1999;518:421-426). This was a large population based, case-control study, including 167 patients with schizophrenia. The findings support the concept of environmental risk factors and prenatal or natal brain damage in some cases of early onset schizophrenia.

These articles and others in the adult literature, concerning the recent evidence for a neurological basis for autism and schizophrenia, are important to pediatric neurologists who will be consulted by child psychiatrists regarding the diagnosis and treatment of underlying neuropathology.

\section{SEIZURE DISORDERS}

\section{AFFECTIVE DISORDERS IN INFANTILE SPASMS}

Facial expression of affect in 28 children with intractable infantile spasms was studied longitudinally for 1.8 years after epilepsy surgery, at the Department of Psychiatry, Mental Retardation Research Center, UCLA, Los Angeles. The mean age at onset of spasms was 2.8 mos, surgery was performed at a mean age of 18 mos, and age at last follow-up was 40 mos. Surgery consisted of hemispherectomy in 11 and multilobar resection in 17. The Maximally Discriminate Movement Coding System (MAX) and Early Social Communication Scale (ESCS) were used for measuring discrete facial movement changes related to emotion. Epilepsy surgery was associated with a significant increase in the use of positive affect (surprise, astonishment, joy), irrespective of seizure, AE drug-related, and surgical variables. Children with a right hemispherectomy did not express more positive or negative affect than those with left hemispherectomy. At base-line and $1.8 \mathrm{yrs}$ after surgery, a low rate of negative affect (sadness, anger, discomfort) was expressed. The lateralization (right hemisphere dominance) theory of emotional expression, and the valence theory (both hemispheres involved- left subserving positive emotion and right controlling negative affect) were not supported. 